\title{
In Vitro Hydroxyapatite-Forming Ability and Antimicrobial Properties of Mesoporous Bioactive Glasses Doped with Ti/Ag
}

\author{
Huiming Lin, Jing Zhang, Fengyu Qu, Jingjie Jiang, and Pingping Jiang \\ Key Laboratory for Photonic and Electronic Bandgap Materials, Ministry of Education and College of \\ Chemistry and Chemical Engineering, Harbin Normal University, Harbin 150025, China
}

Correspondence should be addressed to Fengyu Qu; qufengyu2010@yahoo.cn

Received 7 December 2012; Revised 24 February 2013; Accepted 3 March 2013

Academic Editor: Zhongkui Hong

Copyright (C) 2013 Huiming Lin et al. This is an open access article distributed under the Creative Commons Attribution License, which permits unrestricted use, distribution, and reproduction in any medium, provided the original work is properly cited.

Mesoporous bioactive glasses (MBGs) doped with Ti/Ag were synthesized. The structural, morphological, and textural properties of all samples were investigated by small-angle X-ray diffraction (XRD), scanning electron microscopy (SEM), Fourier transform infrared (FTIR) spectroscopy, and $\mathrm{N}_{2}$ adsorption-desorption technique. In vitro hydroxyapatite- (HAP-) induced growth and antimicrobial properties of these materials were investigated in detail. These results indicate that MBGs-Ti/Ag possess faster HAPinduced growth and higher sterilization rate than the pure MBGs materials. With Ti/Ag doping, these functional MBGs show the improved characteristics and have more promising potential in bone-tissue regeneration systems and surgery.

\section{Introduction}

With the speedy increase of the aged population, the numbers of osteoporotic patients are increasing. The research on bone repair and replacement becomes the research focus $[1,2]$. Among a variety of materials for bone regeneration, bioactive glasses (BGs) have attracted wide attention since the first report by Hench et al. [3]. When implanted into the human body, BGs could form chemical bonds with living bone tissue and promote the bone repair speedily and effectively. Meanwhile, BGs also have excellent biocompatibility and selfdegradation [4-6]. With regular pore size, high pore volume, larger surface area, and uniform component, mesoporous BGs (MBGs) improve the property for bone regeneration. It was reported that the increase of the specific surface area and pore volume of MBGs may apparently accelerate the kinetic deposition of HAP $[7,8]$. Later, some reports testify that the component of materials also plays the significant role in the bone regeneration. The doping method was usually used to enhance the property of these implants. For example, Elena Landi groups used Sr-substituted hydroxyapatites for osteoporotic bone replacement with enhanced repair ability [9]. Ozturk group found that the friction coefficient and specific wear rate could be increased obviously after $\mathrm{MgO}$ and $\mathrm{F}$ doping in BGs [10].

In bone reconstruction surgeries, there are two influence factors that should be considered: (1) chemical bond with living bone; (2) without bacteria infection [11-13]. As we know, MBGs show well chemical bond with living bone. The doping of antimicrobials improves the antibacterial property of MBGs which is very important in practical applications. Silver series and titanium series are the common antimicrobials. $\mathrm{Ag}$ is one of the most important antibacterial materials due to the excellent antimicrobial properties of $\mathrm{Ag}$ and $\mathrm{Ag}+$, which are particularly significant for being associated with biomaterials and are extensively used for infections wound reparation $[14,15]$. Titanium series antimicrobial agent is a typical undissolved antimicrobial agent $[16,17]$. In addition, $\mathrm{TiO}_{2}$ has received more attentions due to well stability, being environmentally benign, safety, and broad-spectrum antibiosis [18]

In this paper, MBGs doped with $\mathrm{Ti}$ (MBGTs) were synthesized by an evaporation-induced self-assembly (EISA) method. And MBG doped with Ag (MBGA) was synthesized by immersed the MBG into the $\mathrm{AgNO}_{3}$ solution, followed by pyrolyzed. In vitro, the apatite forming ability and antimicrobial properties of these materials were investigated in detail. 


\section{Experimental Procedure}

2.1. Materials. All the chemicals were purchased from commercial source and used as received without further purification. Chemicals detailed list is triblock polyethylene oxidepropylene oxide block copolymer (P123, average molecular weight: 5800, Aldrich), tetraethyl orthosilicate (TEOS, Tiantai Chemical Co., Tianjin), triethyl phosphate (TEP, Xinxi reagent Co., Shenyang), $\mathrm{Ca}\left(\mathrm{NO}_{3}\right)_{2} \cdot 4 \mathrm{H}_{2} \mathrm{O}$ (Guangfu Industry of Fine Chemicals Institute, Tianjin), ethanol (EtOH, Yongda Chemical Reagent Company, Tianjin), tetrabutyl titanate $\left(\left[\mathrm{CH}_{3}\left(\mathrm{CH}_{2}\right)_{3} \mathrm{O}\right]_{4} \mathrm{Ti}\right.$, Guangfu Industry of Fine Chemicals Institute, Tianjin), silver nitrate $\left(\mathrm{AgNO}_{3}\right.$, Research Institution of Fine Chemicals, Shanghai), Agar (Bacteriological Grade, Japan), Tryptone (Aoboxing Biotechnology Company, Beijing), and yeast extract (Basing stoke Oxold LTD, England).

2.2. Synthesis of $M B G, M B G T$, and $M B G A$. MBG has been synthesized using P123 as the structure directing agent. In a typical synthesis, $4.0 \mathrm{~g}$ of P123, $0.73 \mathrm{~g}$ of TEP, $6.7 \mathrm{~g}$ of TEOS, $1.4 \mathrm{~g}$ of $\mathrm{Ca}\left(\mathrm{NO}_{3}\right)_{2} \cdot 4 \mathrm{H}_{2} \mathrm{O}$, and $1 \mathrm{~g}$ of $\mathrm{HCl}$ solution $(0.5 \mathrm{M})$ were dissolved in $60 \mathrm{~g}$ of ethanol and stirred at room temperature for $1 \mathrm{D}$. The resulting sol was introduced into a Petri dish to undergo an EISA process. The dried gels were calcined at $973 \mathrm{~K}$ for $5 \mathrm{~h}$ to remove the template.

The synthesis of MBGT is similar to the synthesis process of MBG. Also, $0.5 \mathrm{~mL}$ of tetrabuty titanate was added into the MBG precursor solution. Then, the solution was stirred at room temperature for $1 \mathrm{D}$. The resulting sol was introduced into a Petri dish to undergo an EISA process. The dried gels were calcined at $973 \mathrm{~K}$ for $5 \mathrm{~h}$ to remove the template. The sample was named as MBGT.

The synthesis of MBGA: $0.5 \mathrm{~g}$ of MBG powders was added into $100 \mathrm{~mL}$ of $0.01 \mathrm{~mol} / \mathrm{L} \mathrm{AgNO}_{3}$ solution and sufficiently stirred for $2 \mathrm{~h}$ in the dark condition. The sample was collected by centrifugation and washed with deionized water for several times, dried at $40^{\circ} \mathrm{C}$ for $12 \mathrm{~h}$ in the dark condition. Finally, the sample was calcined at $723 \mathrm{~K}$ for $4 \mathrm{~h}$. The sample was named as MBGA. These materials were pestled by mortar and become to powders that were used to conduct experiments.

\subsection{In Vitro Apatite-Forming Ability of $M B G, M B G T$, and} $M B G A$. An amount of $0.5 \mathrm{~g}$ of samples powders was soaked in $300 \mathrm{~mL}$ of simulate body fluid (SBF) at $37^{\circ} \mathrm{C}$. SBF solution has the similar composition and ionic concentration to those of the human plasma. A small quantity of powders was taken out from SBF solution at interval time. Scanning electron microscopy was used to study the surface transformation of the samples.

\subsection{Antimicrobial of $M B G, M B G T$, and $M B G A$}

(1) Preparation of culture medium. In a typical method, $6 \mathrm{~g}$ of yeast extract, $10 \mathrm{~g}$ of tryptone, $5 \mathrm{~g}$ of $\mathrm{NaCl}$, and $15 \mathrm{~g}$ of agar were dissolved in $1000 \mathrm{~mL}$ of deionized water. Then, culture medium was sterilized for $1 \mathrm{~h}$ at $121^{\circ} \mathrm{C}$. Then, the culture medium was cooled for

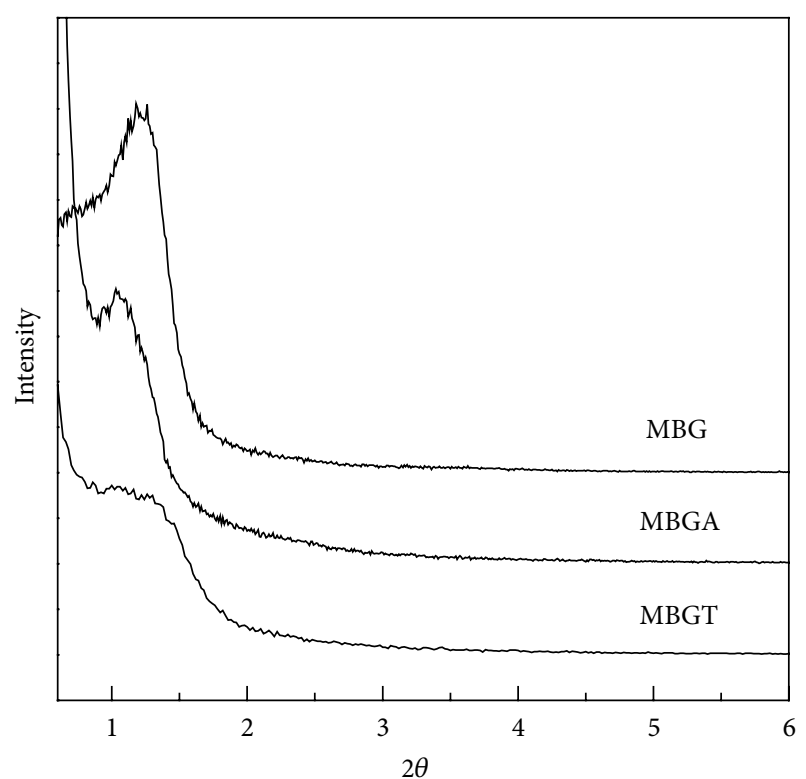

FIGURE 1: Small-angle XRD patterns of MBG, MBGT, and MBGA.

$30 \mathrm{~min}$, and then transferred to culture dishes at germfree condition.

(2) Antimicrobial test: After comparing tests to choose the best concentration of $\mathrm{E}$. coli, the best quality of the materials and test conditions, the optimal test concentration is $1.6 \mu \mathrm{L}$ of Escherichia coli was dissolved in $50 \mathrm{~mL}$ of physiological saline. Also $2 \mathrm{mg}$ of MBG, MBGT, and MBGA were added into $1 \mathrm{~mL}$ of diluent colibacillus solution, respectively. Then, the samples were cultivated for 1 hour in $37^{\circ} \mathrm{C}$ at table concentrator. The samples were centrifuged at $1500 \mathrm{r} / \mathrm{min}$ for $5 \mathrm{~min}$, and then $20 \mu \mathrm{L}$ of superstratum clear liquid were homogeneous spread on culture medium, respectively. The culture dishes were cultivated for 24 hours in $37^{\circ} \mathrm{C}$ constant temperature incubator. After $1 \mathrm{D}$, the colony amounts were counted out and took a picture, to determine the bactericidal capacity of the materials by the number of bacterial colonies on the Petri dish after 24 hours. Calculation of kill rate for colibacillus is based on the following equation (1):

$$
w \%=\frac{\left(N_{0}-N\right)}{N_{0}} \times 100,
$$

where $N_{0}$ is the amount of survived colibacillus of blank sample, and $N$ is the amount of survived colibacillus number of the mesoporous material sample.

2.5. Characterization. Samples were characterized by Scanning Electron Microscope (SEM, Hitachi S-4800). XRD data were collected on SIEMENSD5005 diffractometer with $\mathrm{CuKa}$, using a radiation at $40 \mathrm{kV}$ and $30 \mathrm{~mA}$. The nitrogen adsorption/desorption, surface areas, and median pore diameters were measured using a Micromeritics ASAP $2010 \mathrm{M}$ 


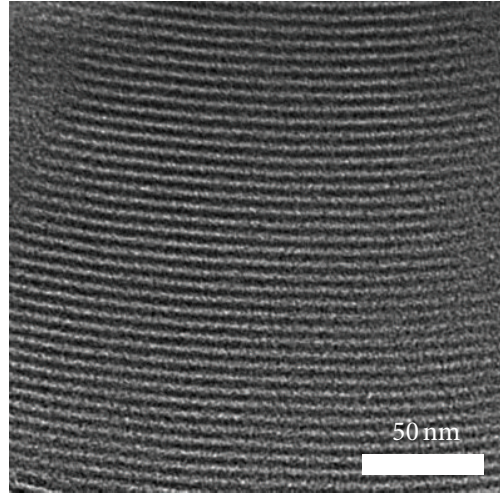

(a)

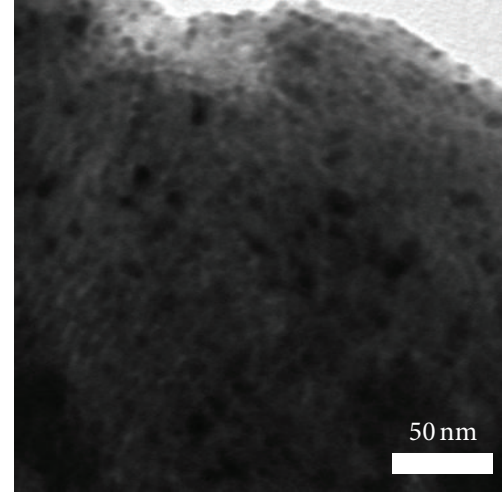

(b)

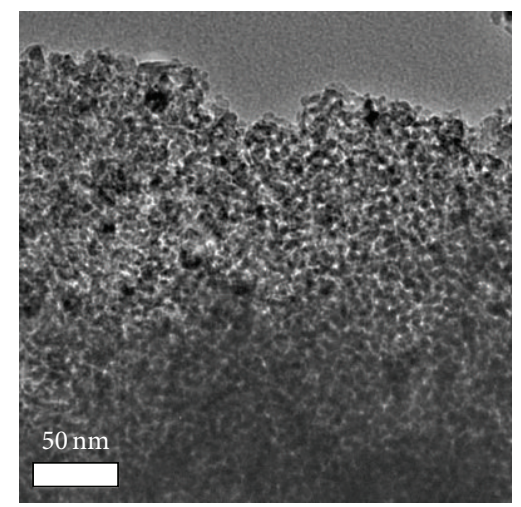

(c)

Figure 2: TEM images of (a) MBG, (b) MBGA, and (c) MBGT.

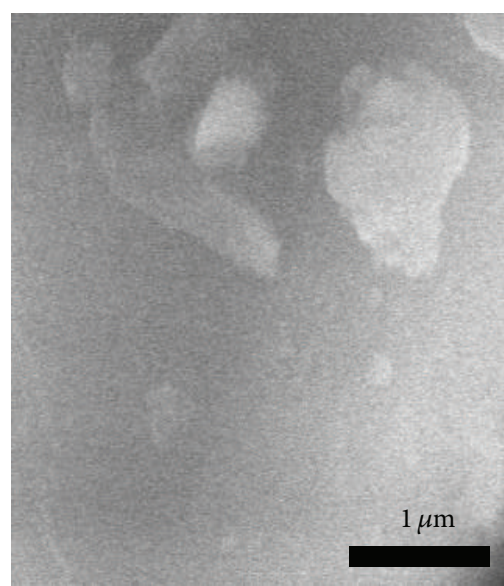

(a)

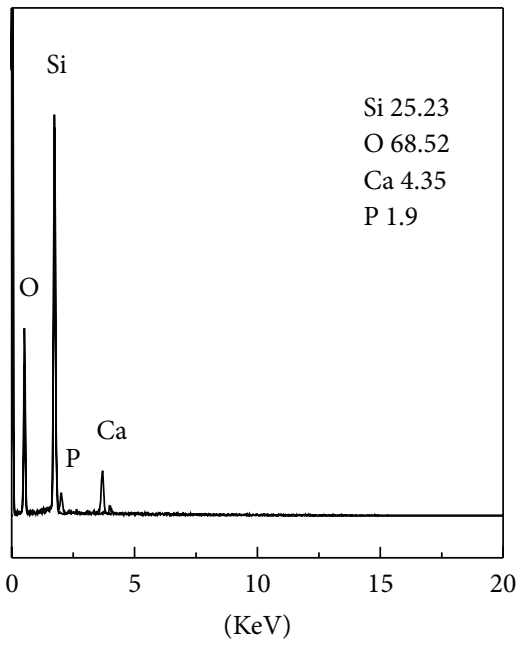

(d)

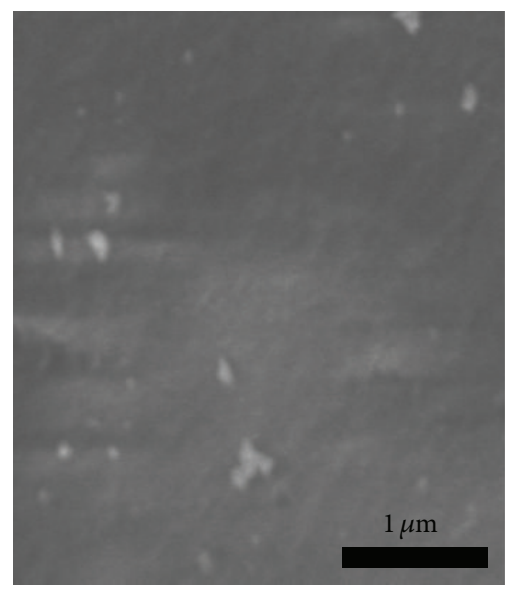

(b)

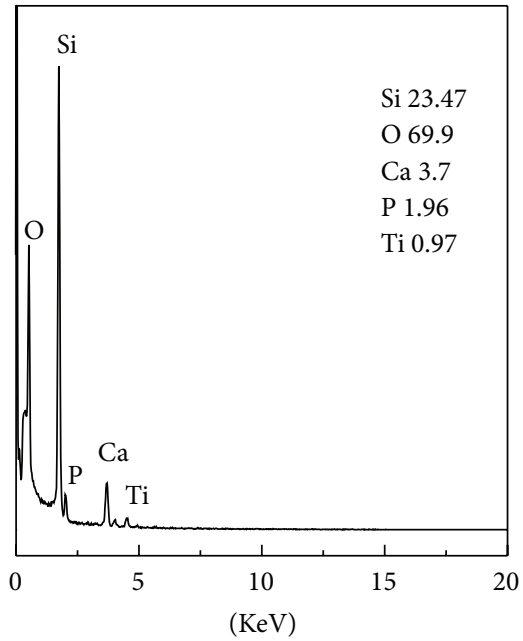

(e)

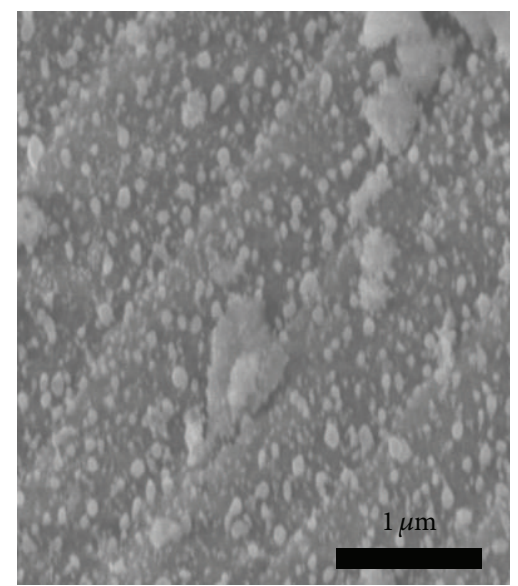

(c)

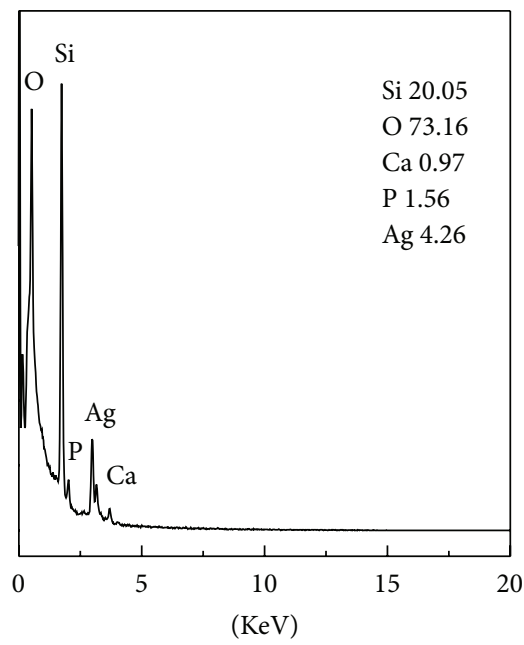

(f)

FIGURE 3: SEM images of the materials: (a) MBG, (b) MBGT, and (c) MBGA; EDS images of the materials: (d) MBG, (e) MBGT, and (f) MBGA. 


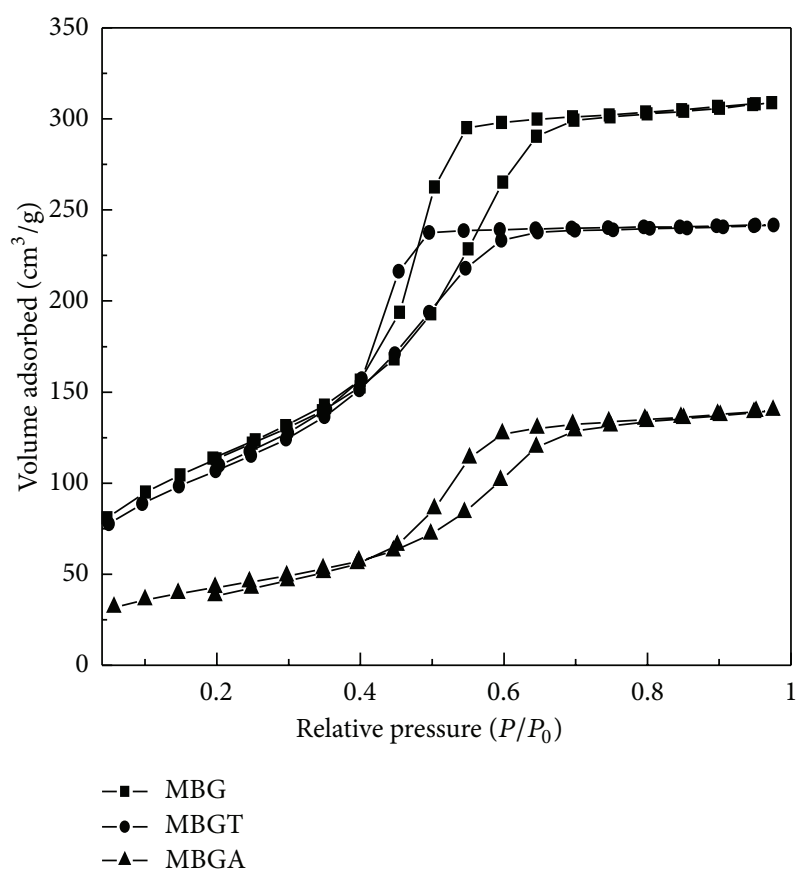

(a)

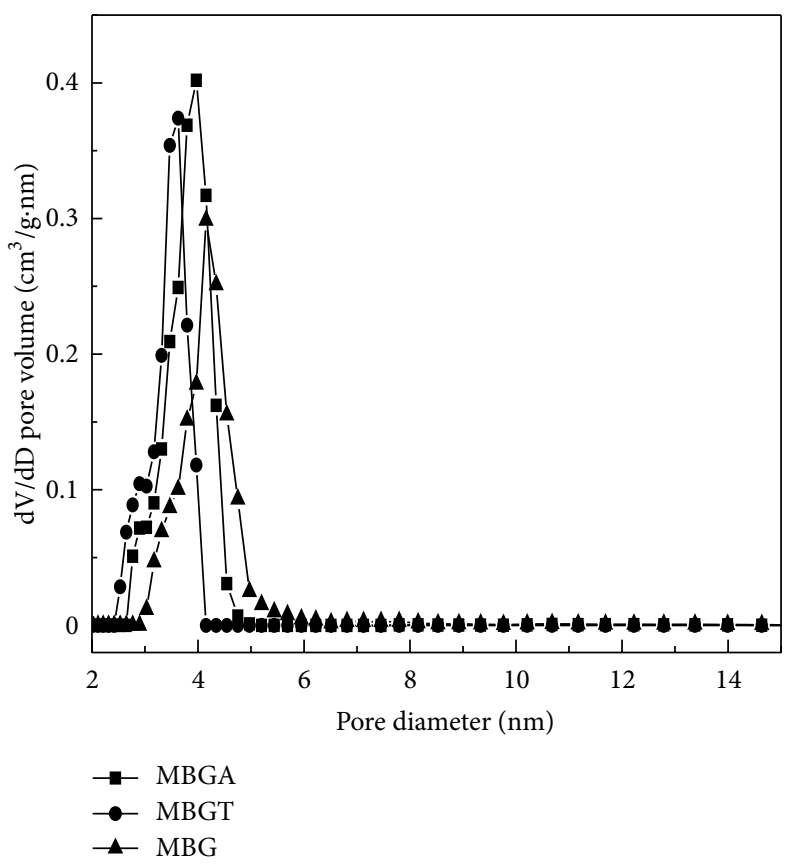

(b)

FIGURE 4: (a) Nitrogen adsorption-desorption isotherm and (b) pore size distribution.

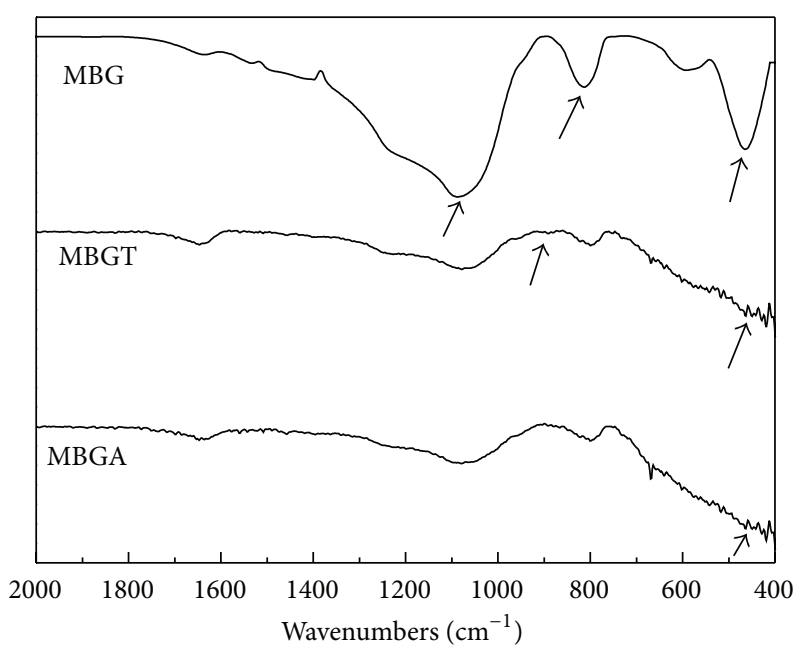

FIGURE 5: FTIR spectra of MBG, MBGT, and MBGA.

sorptometer. Before measured at $77 \mathrm{~K}$, the samples were degassed at $373 \mathrm{~K}$ for $12 \mathrm{~h}$. Specific surface areas and pore size distributions were calculated using the Brunauer-EmmettTeller (BET) and Barrett-Joyner-Halenda (BJH) models from the adsorption branches, respectively. An FTIR spectrometer (JASCOFT/IR-420) was used to record infrared spectra of the mesoporous materials by the $\mathrm{KBr}$ method. The powders samples were pressed onto a tungsten mesh grid and installed in an in situ FTIR transmission cell, and the samples were outgassed in a vacuum system with a residual pressure of less than $3 \times 10^{-4}$ Torr at ambient temperature.

\section{Results and Discussion}

3.1. Characterization of Samples. Figure 1 shows the smallangle XRD patterns of the three mesoporous glasses. As shown in Figure 1, the small-angle XRD pattern of MBG has a diffraction peak at $2 \theta=1.23^{\circ}$, which can be indexed to the (100) diffraction of the two-dimensional hexagonal mesostructure [19]. After the Ti and Ag doping, the peak decreases obviously, implying the less ordered mesoporous structure of MBGT and MBGA. And the MBGT possesses the weakest diffraction peak (Figure 1), because of the quick hydrolyzation of tetrabuty titanate that destroys the ordered degree of MBGT. The structure of these samples also can be conformed from TEM images (Figure 2). From Figure 2(a), MBG shows the ordered straight porous structure. MBGA also shows the ordered straight structure with a lot of dark points, ascribing to the Ag nanoparticles. However, MBGT just possesses the worm-like structure that is consistent with the result of XRD.

The morphology and components of MBG, MBGT, and MBGA are exhibited in Figure 3. It can be observed that all the samples reveal block morphology (Figures 2(a)-2(c)), and MBG and MBGT possess smooth surface (Figures 3(a) and 3(b)). However, there are many small particles on the surface of MBGA (Figure 3(c)). The components of three mesoporous glasses have been investigated by EDS, and all element components were shown in Figures 3(d)-3(f). From Figures 3(d)-3(f), it is clearly revealed that Ti and Ag have been doped in MBGs successfully. The atom percent of the two elements is about $0.97 \%(\mathrm{Ti})$ and $4.26 \%(\mathrm{Ag})$, respectively.

The $\mathrm{N}_{2}$ adsorption-desorption isotherms and pore size distribution curves of the samples are shown in Figure 4. The 


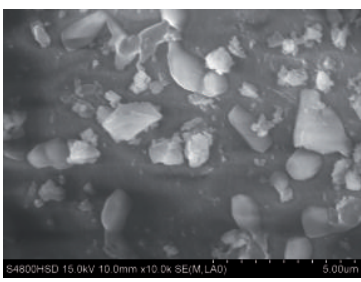

(a)

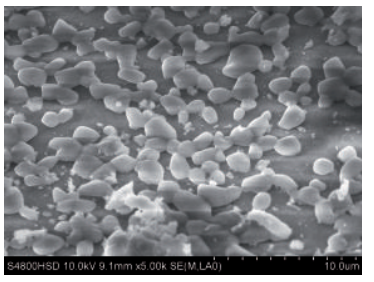

(e)

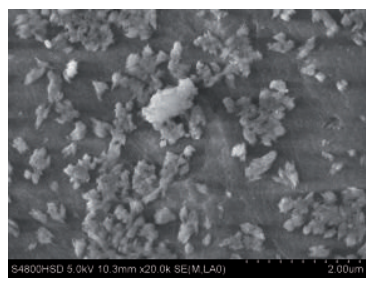

(i)
$6 \mathrm{~h}$

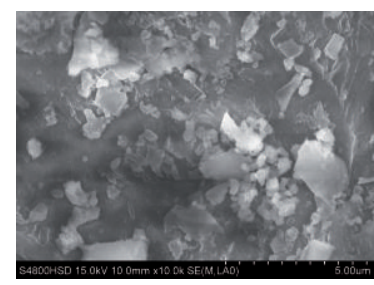

(b)

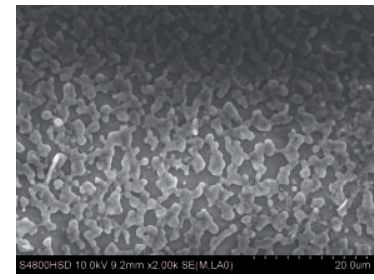

(f)

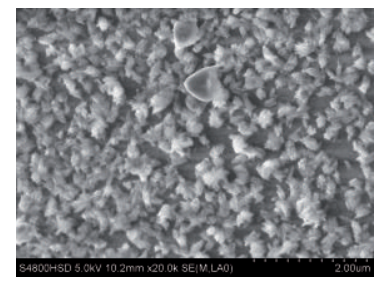

(j)
$9 \mathrm{~h}$

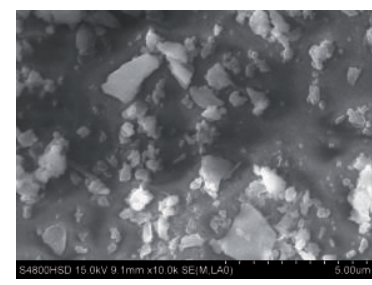

(c)

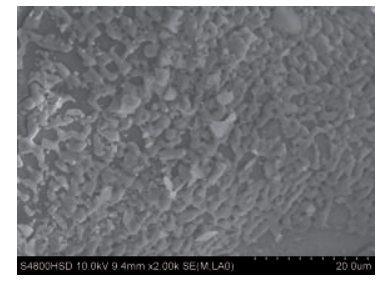

(g)

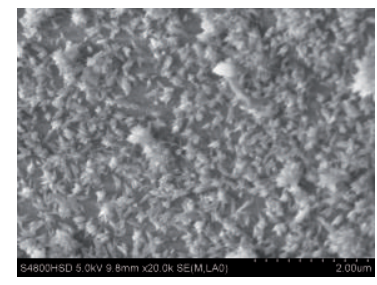

(k)
$12 \mathrm{~h}$

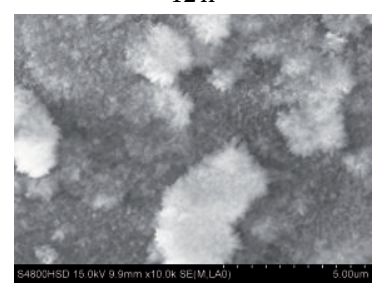

(d)

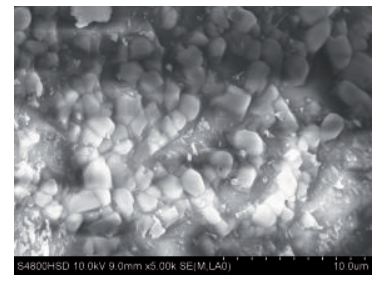

(h)

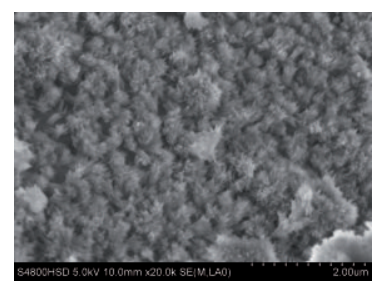

(1)

FIGURE 6: SEM images of the surface of the samples after soaking in SBF for various periods: ((a)-(d)) MBG, ((e)-(h)) MBGT, and ((i)-(l)) MBGA.

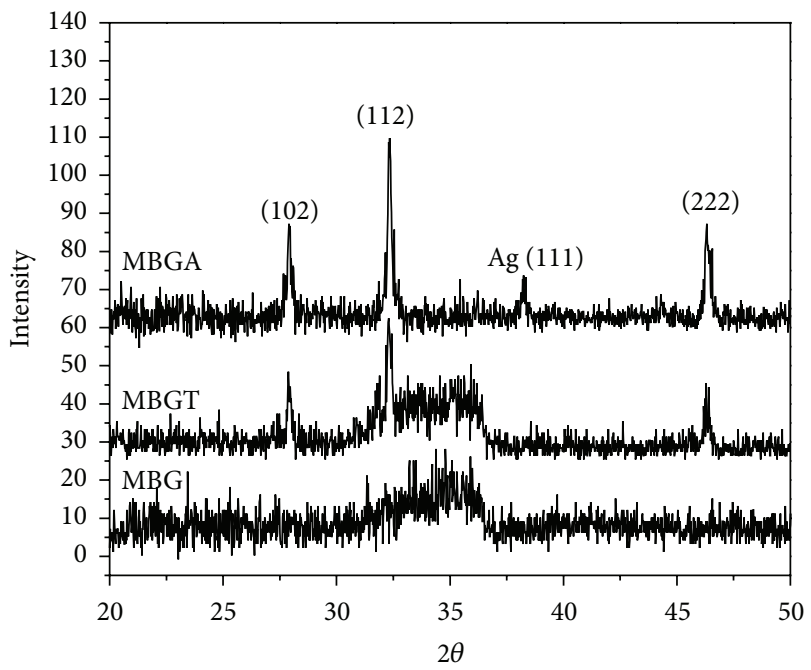

FIGURE 7: Large- angle XRD of the surfaces of samples after soaking in SBF for 1 day.

nitrogen adsorption-desorption isotherms of MBG, MBGT, and MBGA (Figure 4(a)) indicate the type IV isotherm with H1-type hysteresis loops of the mesoporous materials with uniform pore size. The surface area, pore volume, and pore size of these samples were listed in Table 1. MBG possesses high pore volume and large specific BET surface area,
TABLE 1: Structure parameters of the samples.

\begin{tabular}{lccc}
\hline & Pore size $(\mathrm{nm})$ & $V_{p}\left(\mathrm{~cm}^{3} / \mathrm{g}\right)$ & $S_{\mathrm{BET}}\left(\mathrm{m}^{2} / \mathrm{g}\right)$ \\
\hline MBG & 4.2 & 0.44 & 371 \\
MBGT & 3.6 & 0.32 & 362 \\
MBGA & 4.0 & 0.30 & 282 \\
\hline
\end{tabular}

$0.44 \mathrm{~cm}^{3} / \mathrm{g}$ and $371 \mathrm{~m}^{2} / \mathrm{g}$. Due to the Ti and Ag doping, the pore volume and specific BET surface area are reduced obviously, $0.32 \mathrm{~cm}^{3} / \mathrm{g}$ and $362 \mathrm{~m}^{2} / \mathrm{g}$, and $0.30 \mathrm{~cm}^{3} / \mathrm{g}$ and $282 \mathrm{~m}^{2} / \mathrm{g}$ for MBGT and MBGA, respectively. The narrow peaks of BJH pore size distribution curves for MBG, MBGT, and MBGA are focused on 4.2, 3.6, and $4.0 \mathrm{~nm}$, respectively (Figure 4(b)). With the fast hydrolysis rate, the addition of tetrabuty titanate disturbed the assembly of the surfactant, making the poor ordered structure and deducing the pore size. MBGA was synthesized by impregnating $\mathrm{AgNO}_{3}$ solution into the pore of MBG. The postdoping method make Ag nanoparticles possess the porous space and decrease pore size. So, MBGA and MBGT show smaller pore size than MBG.

Figure 5 shows the FT-IR spectra of MBG, MBGT, and MBGA. It can be found that there are three characteristic absorption bands at 1085,815 , and $465 \mathrm{~cm}^{-1}$, which are attributed to $\mathrm{Si}-\mathrm{O}-\mathrm{Si}$ asymmetric stretching vibration, $\mathrm{Si}-\mathrm{O}-\mathrm{Si}$ symmetric stretching vibration, and $\mathrm{Si}-\mathrm{O}$ bending vibration for MBG [20,21]. Compared with the FT-IR spectra of MBG, the FT-IR spectra of MBGT and MBGA were also changed. 


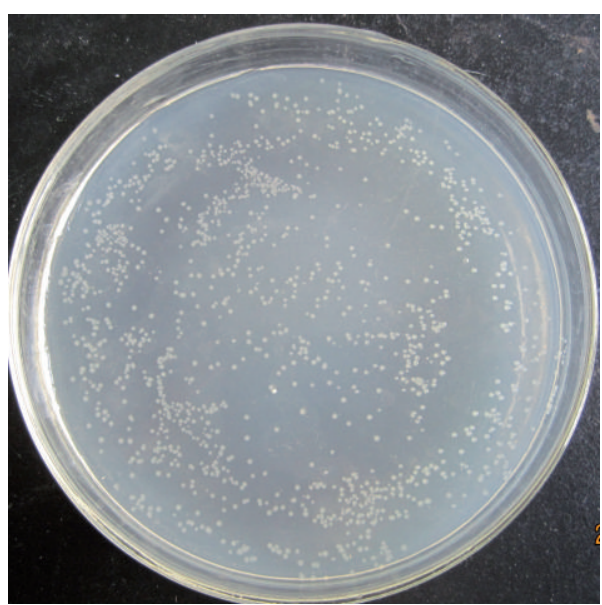

(a)

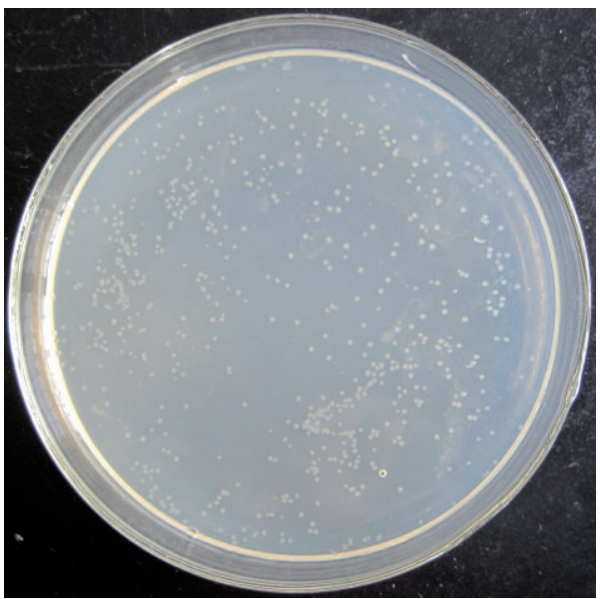

(c)

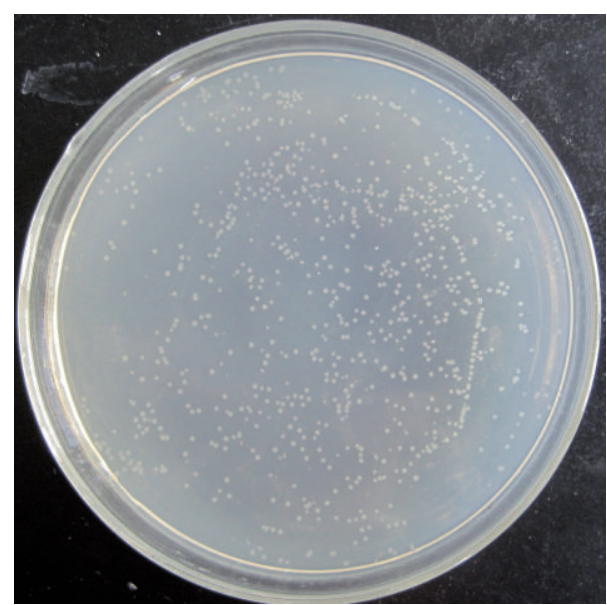

(b)

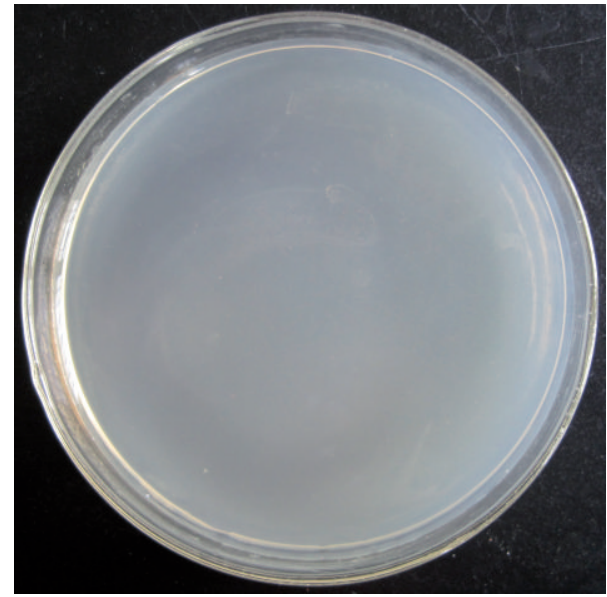

(d)

FIGURE 8: Photos of colibacillus grow status: (a) blank samples, (b) MBG, (c) MBGT, and (d) MBGA.

After doped $\mathrm{Ti}$ and $\mathrm{Ag}$, those interactions between the $\mathrm{Ti} / \mathrm{Ag}$ with $\mathrm{Si}-\mathrm{OH}$ make $\mathrm{Si}-\mathrm{O}-\mathrm{Si}$ symmetric stretching vibration weak and $\mathrm{Si}-\mathrm{O}$ bending vibration unclear. The characteristics absorption band at $895 \mathrm{~cm}^{-1}$ are attributed to Ti-O for MBGT. MBGA has no characteristic absorption band of Ag$\mathrm{O}$ stretching vibration in the FT-IR spectra testifying without silver oxide.

3.2. Apatite-Forming Ability of $M B G, M B G T$, and $M B G A$. The SEM images in Figure 6 indicate the formation of apatite on the surface of these functional MBGs samples in SBF solution for a period of time. It only takes $9 \mathrm{~h}$ and $6 \mathrm{~h}$ for MBGT and MBGA to form an HAP layer on their surface, which is faster than that of MBG (12 h).

Figure 7 shows the large-angle XRD patterns of these materials after soaking in SBF for 1 day. All samples show the typical diffraction of HAP. The peak strength of MBGA is strongest of all, later is MBGT, and the BMG is the lowest. MBGA and MBGT show the faster HAP formation ability than pure MBG. When MBGT and MBGA were immersed into SBF solution, plenty of new $\mathrm{Si}-\mathrm{OH}$ was quickly formed on the surfaces of materials, because the doped component was dissolved into SBF solution. That induces abundant of HAP nucleation to improve the formation of HAP [22]. In addition, the Ti and $\mathrm{Ag}$ ion concentration of the solution is $0.00012 \mathrm{mg} / \mathrm{L}$ and $0.014 \mathrm{mg} / \mathrm{L}$ (from ICP), which are all under safe concentration. And the dissolution of $\mathrm{Ag}$ is the fastest $(0.014 \mathrm{mg} / \mathrm{L})$, most new $\mathrm{Si}-\mathrm{OH}$ were formed, inducing the fastest HAP formation ability. From the previous investigation, the doping is also useful for the HAP formation.

3.3. Antimicrobial Test of $M B G, M B G T$, and $M B G A$. Figures 8 and 9 exhibit the antimicrobial property of these samples. The colibacillus amounts were counted out and the kill rates were calculated through (1). Each value in the result is the average of the results of three parallel experiments. Figure 8(b) was drawn through the results calculated by (1). It clearly indicates that these materials have the effective antimicrobial ability. Kill rates for colibacillus of MBG, MBGT, and MBGA reach $30.75,49.71$, and $99.80 \%$, respectively. MBGA has the highest sterilization ability. Pure MBG shows some kill rate ability and bacterial adhesion activity [23]. When doped with $\mathrm{Ti}$ 


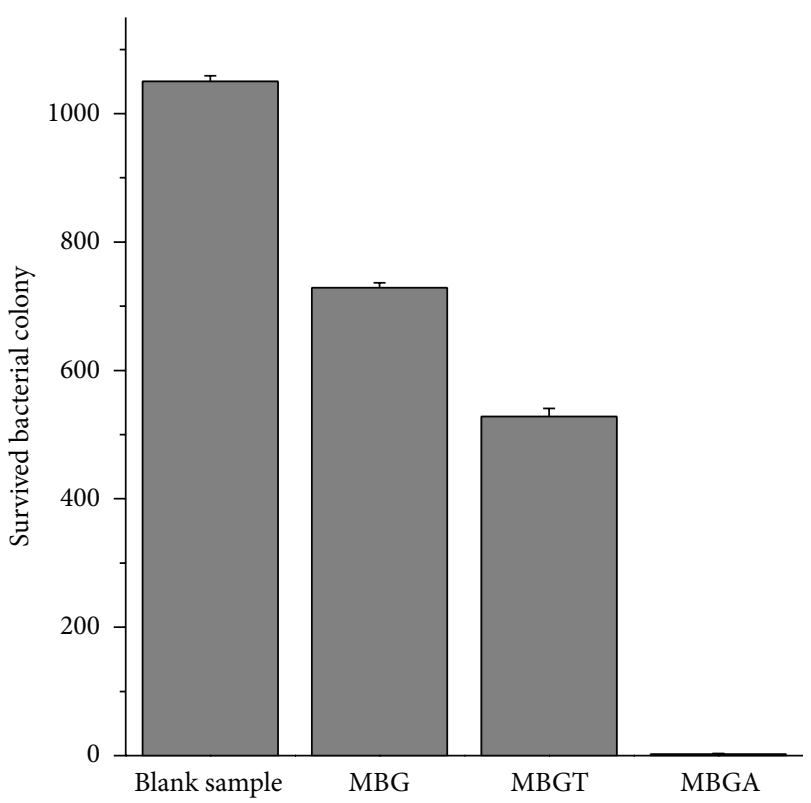

(a)

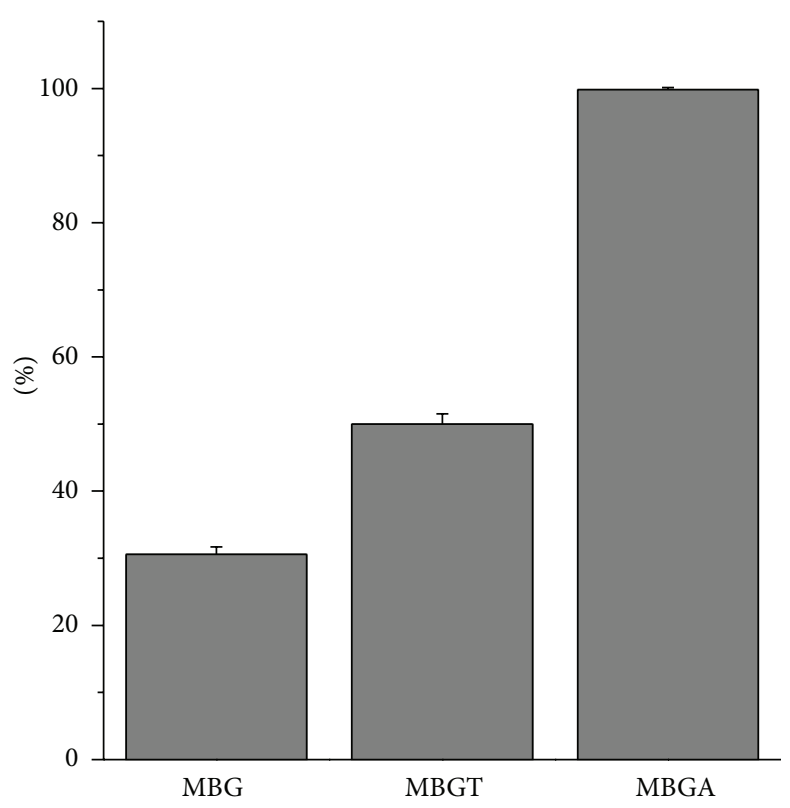

(b)

FIGURE 9: The survived bacterial colony of colibacillus (a) and sterilization rate (b) of MBG, MBGT, and MBGA. The results represent the means \pm SDs $(n=4)$.

and Ag, their sterilization ability increased obviously. The main reasons are the release of ${ }^{-} \mathrm{OH}$ free radicals which have stronger oxidizability from MBGT. Organic matters could be resolved by the ${ }^{-} \mathrm{OH}$ free radical on the surface of MBGT, and the procreant endotoxin was also resolved by ${ }^{-} \mathrm{OH}$ free radical after bacteria were died out. And nano-Ag would ionization out Ag ion on the surface for MBGA. Then, Ag ion could get in touch with colibacillus and act on protein and enzyme of colibacillus which are live require, then influence their breeding metabolism. Above all, the Ti and Ag doping mesoporous bioactive glasses have excellent kill rate ability.

\section{Conclusions}

MBGA and MBGT were synthesized using the simple method. The HAP-forming ability and antimicrobial properties of these functional MGBs were investigated in detail. Because of the $\mathrm{Ti}$ and $\mathrm{Ag}$ doping, MBGA and MBGT possess the faster HAP-forming ability and higher kill rate than the conventional MBG. These functional MBGs have outstanding properties and may become promising candidate for bone-tissue regeneration systems and surgery operation.

\section{Acknowledgments}

Financial support for this study was provided by Foundation of Harbin Educational Committee (12521164).

\section{References}

[1] Y. Y. Yan, L. Shirley, C. Y. Lu, and C. Céline, "Bone morphogenetic protein 2 stimulates endochondral ossification by regulating periosteal cell fate during bone repair," Bone, vol. 47, no. 1, pp. 65-73, 2010.
[2] X. F. Li, J. J. Jiang, Y. Wang, X. Nie, and F. Y. Qu, "Preparation of multilevel macroporous materials using natural plants as templates," Journal of Sol-Gel Science and Technology, vol. 56, no. 1, pp. 75-81, 2010.

[3] L. L. Hench, R. Splinter, W. Allen, and T. Greenlee, "Bonding mechanisms at the interface of ceramic prosthetic materials," Journal of Biomedical Materials Research, vol. 5, no. 6, pp. 117141, 1971.

[4] D. Arcos, M. Vila, A. López-Noriega et al., "Mesoporous bioactive glasses: mechanical reinforcement by means of a biomimetic process," Acta Biomaterialia, vol. 7, no. 7, pp. 29522959, 2011.

[5] C. T. Kresge, M. E. Leonowicz, W. J. Roth, J. C. Vartuli, and J. S. Beck, "Ordered mesoporous molecular sieves synthesized by a liquid-crystal template mechanism," Nature, vol. 359, no. 6397, pp. 710-712, 1992.

[6] M. Vallet-Regi, "Ceramics for medical applications," Journal of the Chemical Society, Dalton Transactions, no. 2, pp. 97-108, 2001.

[7] J. Schnieders, U. Gbureck, R. Thull, and T. Kissel, "Controlled release of gentamicin from calcium phosphate-poly(lactic acidco-glycolic acid) composite bone cement," Biomaterials, vol. 27, no. 23 , pp. 4239-4249, 2006.

[8] W. Xia and J. Chang, "Well-ordered mesoporous bioactive glasses (MBG): a promising bioactive drug delivery system," Journal of Controlled Release, vol. 110, no. 3, pp. 522-530, 2006.

[9] E. Landi, A. Tampieri, G. Celotti, S. Sprio, M. Sandri, and G. Logroscino, "Sr-substituted hydroxyapatites for osteoporotic bone replacement," Acta Biomaterialia, vol. 3, no. 6, pp. 961-969, 2007.

[10] J. Park, G. Pekkan, and A. Ozturk, "Wear of MgO-CaO$\mathrm{SiO}_{2}-\mathrm{P}_{2} \mathrm{O}_{5}-\mathrm{F}$-based glass ceramics compared to selected dental ceramics," Research Letters in Materials Science, vol. 2007, Article ID 69897, 5 pages, 2007. 
[11] J. Ye, P. X. Gong, P. Z. Zhi, J. X. Jing, L. Y. Jing, and H. P. Shang, "BMP-2/PLGA delayed-release microspheres composite graft, selection of bone particulate diameters, and prevention of aseptic inflammation for bone tissue engineering," Annals of Biomedical Engineering, vol. 38, no. 3, pp. 632-639, 2010.

[12] T. J. Webster, A. A. Patel, M. N. Rahaman, and B. Sonny Bal, "Anti-infective and osteointegration properties of silicon nitride, poly(ether ether ketone), and titanium implants," Acta Biomaterialia, vol. 8, pp. 4447-4454, 2012.

[13] J. Baas, B. Elmengaard, T. B. Jensen, T. Jakobsen, N. T. Andersen, and K. Soballe, "The effect of pretreating morselized allograft bone with rhBMP-2 and/or pamidronate on the fixation of porous Ti and HA-coated implants," Biomaterials, vol. 29, no. 19, pp. 2915-2922, 2008.

[14] K. Peter, S. Emma, E. A. Ragnhild, C. György, M. Dane, and K. Sigbritt, "Antimicrobial properties of $\mathrm{Ag}^{+}$loaded zeolite polyester polyurethane and silicone rubber and long-term properties after exposure to in-vitro ageing," Polymer Degradation and Stability, vol. 95, no. 9, pp. 1456-1465, 2010.

[15] N. J. Coleman, A. H. Bishop, S. E. Booth, and J. W. Nicholson, " $\mathrm{Ag}^{+}$- and $\mathrm{Zn}^{2+}$-exchange kinetics and antimicrobial properties of 11 Å tobermorites," Journal of the European Ceramic Society, vol. 29, no. 6, pp. 1109-1117, 2009.

[16] H. R. Rong, B. Zhao, and H. C. Gu, "Review on inorganic antibacterial agents," ChemicalWorld, vol. 7, p. 339, 2000.

[17] A. Fujishima and T. N. Ral, "Interfacial photochemistry: fundamental and applications," Pure and Applied Chemistry, vol. 70, no. 1, pp. 2177-2187, 1998.

[18] V. Stranak, H. Wulff, H. Rebl et al., "Deposition of thin titanium-copper films with antimicrobial effect by advanced magnetron sputtering methods," Materials Science and Engineering C, vol. 31, no. 7, pp. 1512-1519, 2011.

[19] G. F. Wei, X. X. Yan, Y. Jing et al., "Synthesis and in-vitro bioactivity of mesoporous bioactive glasses with tunable macropores," Microporous and Mesoporous Materials, vol. 143, no. 1, pp. 157-165, 2011.

[20] J. C. Ro and I. J. Chung, "Structures and properties of silica gels prepared by the sol-gel method," Journal of Non-Crystalline Solids, vol. 130, no. 1, pp. 8-17, 1991.

[21] G. Orcel, J. Phalippou, and L. L. Hench, "Structural changes of silica xerogels during low temperature dehydration," Journal of Non-Crystalline Solids, vol. 88, no. 1, pp. 114-130, 1986.

[22] N. Miyata, K. I. Fuke, Q. Chen, M. Kawashita, T. Kokubo, and T. Nakamura, "Apatite-forming ability and mechanical properties of PTMO-modified $\mathrm{CaO}-\mathrm{SiO}_{2}-\mathrm{TiO}_{2}$ hybrids derived from solgel processing," Biomaterials, vol. 25, no. 1, pp. 1-7, 2004.

[23] J. K. Teemu, E. Jaime, G. B. Enrique et al., "Bacterial adherence to $\mathrm{SiO}_{2}$-based multifunctional bioceramics," Journal of Biomedical Materials Research A, vol. 89, no. 1, pp. 215-223, 2007. 

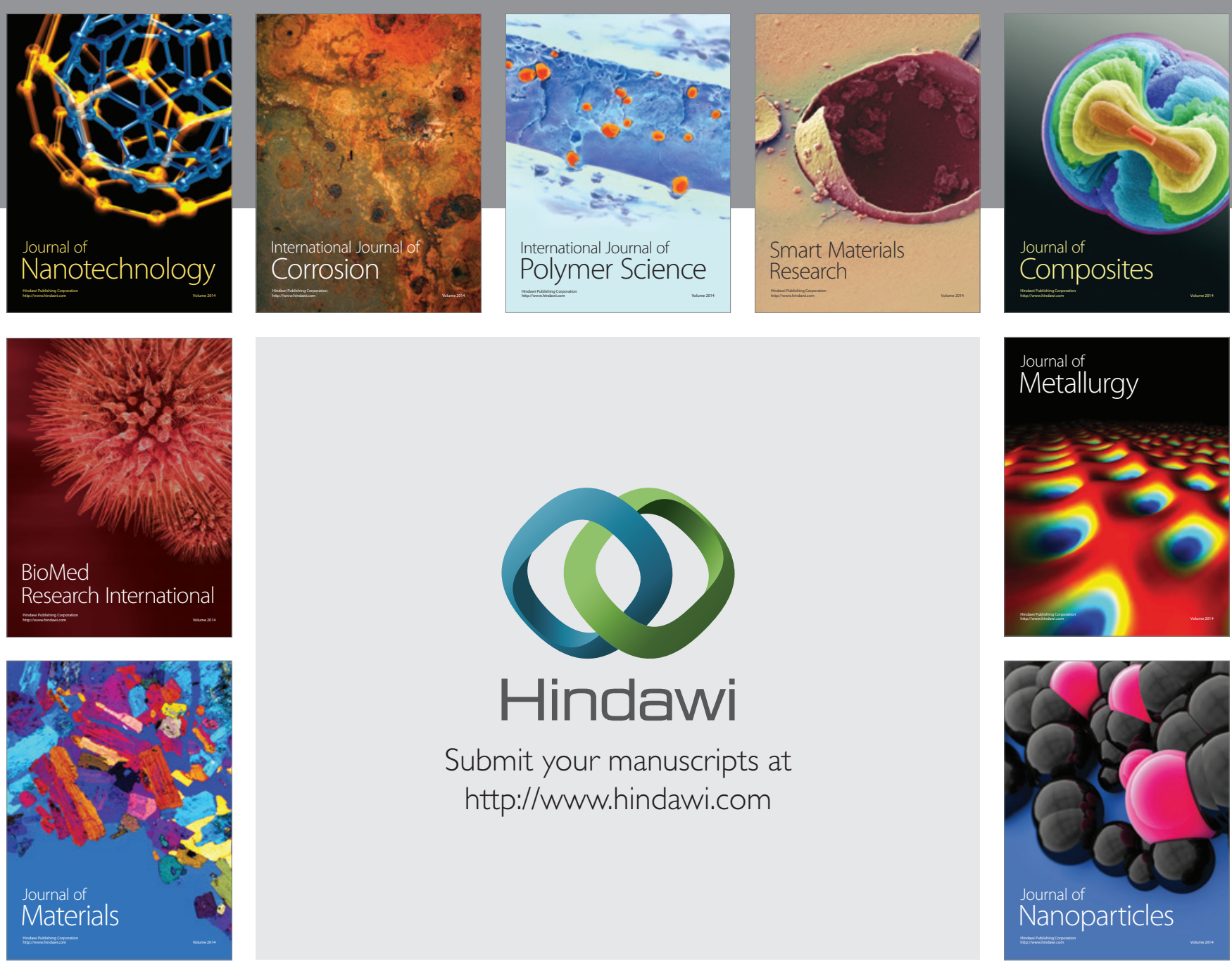

Submit your manuscripts at http://www.hindawi.com
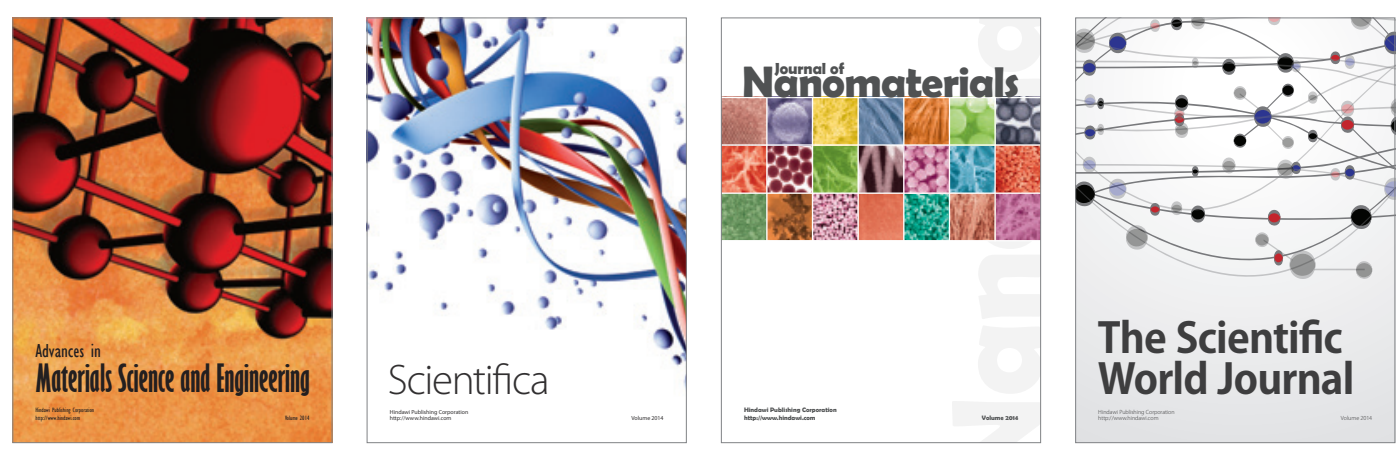

\section{The Scientific World Journal}
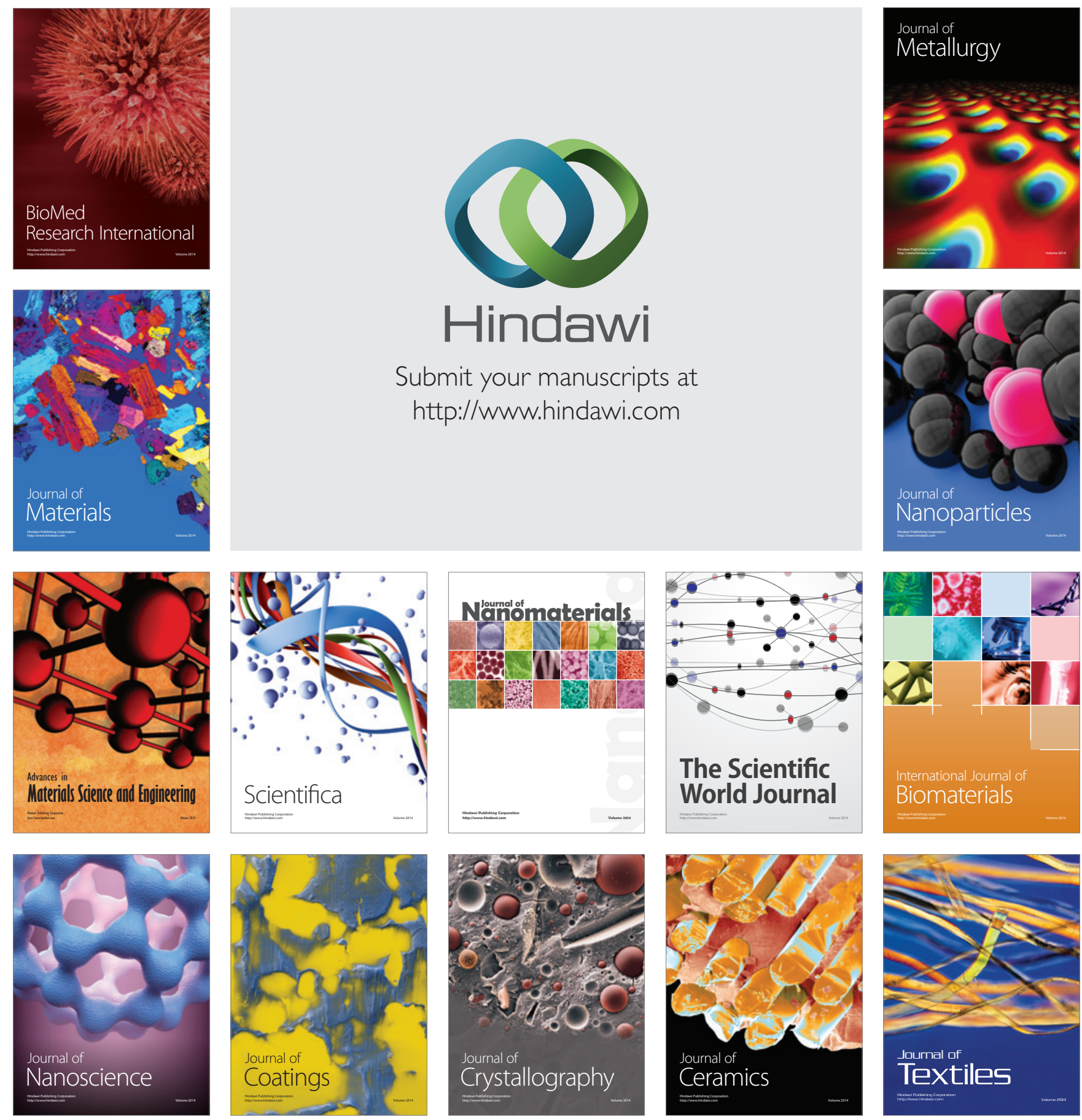